\title{
THE $a$-NUMBERS OF JACOBIANS OF SUZUKI CURVES
}

\author{
HOLLEY FRIEDLANDER, DEREK GARTON, BETH MALMSKOG, RACHEL PRIES, \\ AND COLIN WEIR
}

(Communicated by Matthew A. Papanikolas)

\begin{abstract}
For $m \in \mathbb{N}$, let $S_{m}$ be the Suzuki curve defined over $\mathbb{F}_{2^{2 m+1}}$. It is well-known that $S_{m}$ is supersingular, but the $p$-torsion group scheme of its Jacobian is not known. The $a$-number is an invariant of the isomorphism class of the $p$-torsion group scheme. In this paper, we compute a closed formula for the $a$-number of $S_{m}$ using the action of the Cartier operator on $H^{0}\left(S_{m}, \Omega^{1}\right)$.
\end{abstract}

\section{INTRODUCTION}

Let $m \in \mathbb{N}, q=2^{2 m+1}$, and $q_{0}=2^{m}$. The Suzuki curve $\mathcal{S}_{m} \subset \mathbb{P}^{2}$ is defined over $\mathbb{F}_{q}$ by the homogeneous equation

$$
W^{q_{0}}\left(Z^{q}+Z W^{q-1}\right)=Y^{q_{0}}\left(Y^{q}+Y W^{q-1}\right) .
$$

This curve is smooth and irreducible with genus $g=q_{0}(q-1)$, and it has exactly one point at infinity [8, Proposition 1.1]. The number of points on the Suzuki curve over $\mathbb{F}_{q}$ is $\# S_{m}\left(\mathbb{F}_{q}\right)=q^{2}+1$; this number is optimal in that it reaches Serre's improvement to the Hasse-Weil bound [8, Proposition 2.1].

In fact, $S_{m}$ is the unique $\mathbb{F}_{q}$-optimal curve of genus $g$ [2. This shows that $S_{m}$ is the Deligne-Lusztig variety of dimension 1 associated with the group $S z(q)=$ ${ }^{2} B_{2}(q)$ [7, Proposition 4.3]. The curve $S_{m}$ has the Suzuki group $S z(q)$ as its automorphism group; the order of $S z(q)$ is $q^{2}(q-1)\left(q^{2}+1\right)$, which is very large when compared with $g$. Because of the large number of rational points relative to their genus, the Suzuki curves provide good examples of Goppa codes [4, Section 4.3], [5], 8].

The $L$-polynomial of $S_{m}$ is $\left(1+\sqrt{2 q} t+q t^{2}\right)^{g}$ [7, Proposition 4.3]. It follows that $S_{m}$ is supersingular for each $m \in \mathbb{N}$. This fact implies that the $\operatorname{Jacobian} \operatorname{Jac}\left(S_{m}\right)$ is isogenous to a product of supersingular elliptic curves and that $\operatorname{Jac}\left(S_{m}\right)$ has no 2 -torsion points over $\overline{\mathbb{F}}_{2}$. However, there are still open questions about $\operatorname{Jac}\left(S_{m}\right)$. In this paper, we address one of these by computing a closed formula for the $a$-number of $\operatorname{Jac}\left(S_{m}\right)$.

The $a$-number is an invariant of the 2-torsion group scheme $\operatorname{Jac}\left(S_{m}\right)[2]$. Specifically, if $\alpha_{2}$ denotes the kernel of Frobenius on the additive group $\mathbb{G}_{a}$, then the $a$-number of $S_{m}$ is $a(m)=\operatorname{dim}_{\bar{F}_{2}} \operatorname{Hom}\left(\alpha_{2}, \operatorname{Jac}\left(S_{m}\right)[2]\right)$. It equals the dimension of the intersection of $\operatorname{Ker}(F)$ and $\operatorname{Ker}(V)$ on the Dieudonné module of $\operatorname{Jac}\left(S_{m}\right)[2]$. Having a supersingular Newton polygon places constraints upon the $a$-number but

Received by the editors November 2, 2011 and, in revised form, November 29, 2011. 2010 Mathematics Subject Classification. Primary 11G20, 14H40; Secondary 14G50. Key words and phrases. Suzuki curve, maximal curve, Jacobian, p-torsion, a-number. The third author was partially supported by NSF grant DMS-11-01712. 
does not determine it. The $a$-number also gives partial information about the decomposition of $\operatorname{Jac}\left(S_{m}\right)$ into indecomposable principally polarized abelian varieties (see Lemma 5.2) and about the Ekedahl-Oort type of $\operatorname{Jac}\left(S_{m}\right)[2]$ (see Section 51).

In Section 4, we prove that the $a$-number of $S_{m}$ is $a(m)=q_{0}\left(q_{0}+1\right)\left(2 q_{0}+1\right) / 6$; see Theorem 4.1. The proof uses the action of the Cartier operator on $H^{0}\left(S_{m}, \Omega^{1}\right)$ as computed in Section 3 .

\section{THE $a$-NUMBER}

Suppose $A$ is a principally polarized abelian variety of dimension $g$ defined over an algebraically closed field $k$ of characteristic $p>0$. For example, $A$ could be the Jacobian of a $k$-curve of genus $g$. Consider the multiplication-by- $p$ morphism $[p]: A \rightarrow A$ which is a finite flat morphism of degree $p^{2 g}$. It factors as $[p]=V \circ F$. Here, $F: A \rightarrow A^{(p)}$ is the relative Frobenius morphism coming from the $p$-power map on the structure sheaf; it is purely inseparable of degree $p^{g}$. The Verschiebung morphism $V: A^{(p)} \rightarrow A$ is the dual of $F$.

The kernel of $[p]$ is $A[p]$, the $p$-torsion of $A$, which is a quasi-polarized $B T_{1}$ group scheme. In other words, it is a quasi-polarized finite commutative group scheme annihilated by $p$, again having morphisms $F$ and $V$. The rank of $A[p]$ is $p^{2 g}$. These group schemes were classified independently by Kraft (unpublished) [10] and by Oort [13. A complete description of this topic can be found in [12] or [13].

Two invariants of (the $p$-torsion of) an abelian variety are the $p$-rank and the $a$-number. The $p$-rank of $A$ is $r(A)=\operatorname{dim}_{\mathbb{F}_{p}}\left(\operatorname{Hom}\left(\mu_{p}, A[p]\right)\right)$, where $\mu_{p}$ is the kernel of Frobenius on the multiplicative group $\mathbb{G}_{m}$. Then $p^{r(A)}$ is the cardinality of $A[p]\left(\overline{\mathbb{F}}_{p}\right)$. The a-number of $A$ is $a(A)=\operatorname{dim}_{k}\left(\operatorname{Hom}\left(\alpha_{p}, A[p]\right)\right)$, where $\alpha_{p}$ is the kernel of Frobenius on the additive group $\mathbb{G}_{a}$. It is well-known that $1 \leq a(A)+$ $r(A) \leq g$. Another definition for the $a$-number is

$$
a(A)=\operatorname{dim}_{\mathbb{F}_{p}}(\operatorname{Ker}(F) \cap \operatorname{Ker}(V)) .
$$

If $X$ is a (smooth, projective, connected) $k$-curve, then the $a$-number of $A=$ $\operatorname{Jac}(X)$ equals the dimension of the kernel of the Cartier operator $\mathcal{C}$ on $H^{0}\left(X, \Omega^{1}\right)$ [11, 5.2.8]. The reason for this is that the action of $\mathcal{C}$ on $H^{0}\left(X, \Omega^{1}\right)$ is the same as the action of $V$ on $V \operatorname{Jac}(X)[p]$. This is the property that we use to calculate the $a$-number $a(m)$ of the Jacobian of the Suzuki curve $S_{m}$.

\section{Regular 1-Forms For the Suzuki Curves}

In this section, we compute the action of the Cartier operator on the vector space of regular 1-forms for the Suzuki curves.

3.1. Geometry of the Suzuki curves. Let $m \in \mathbb{N}, q=2^{2 m+1}$, and $q_{0}=2^{m}$. Consider the Suzuki curve $\mathcal{S}_{m} \subset \mathbb{P}^{2}$ defined over $\mathbb{F}_{q}$ by the homogeneous equation

$$
W^{q_{0}}\left(Z^{q}+Z W^{q-1}\right)=Y^{q_{0}}\left(Y^{q}+Y W^{q-1}\right) .
$$

The curve $S_{m}$ is smooth and irreducible and has one point $P_{\infty}$ at infinity (when $W=Y=0$ and $Z=1$ ). Consider the irreducible affine model of $S_{m}$ defined by the equation

$$
z^{q}+z=y^{q_{0}}\left(y^{q}+y\right)
$$

where $y:=Y / W$ and $z:=Z / W$. 
The following result is well-known; see e.g., 8, Proposition 1.1]. We include an alternative proof that illustrates the geometry of some of the quotient curves of $S_{m}$ and an important point about the $a$-number.

Lemma 3.1. The curve $S_{m}$ has genus $g=q_{0}(q-1)$.

Proof. The set $\mathbb{F}_{q}^{*}=\left\{\mu_{1}, \ldots, \mu_{q-1}\right\}$ can be viewed as a set of representatives for the $q-1$ cosets of $\mathbb{F}_{2}^{*}$ in $\mathbb{F}_{q}^{*}$. The Suzuki curve has affine equation $z^{q}-z=f(y)$, where $f(y)=y^{q_{0}+q}+y^{q_{0}+1} \in \mathbb{F}_{2}(y)$. For $1 \leq i \leq q-1$, let $Z_{i}$ be the Artin-Schreier curve with equation $z_{i}^{2}-z_{i}=\mu_{i} f(y)$. As seen in [3, Proposition 1.2], the set $\left\{Z_{i} \rightarrow \mathbb{P}_{y}^{1} \quad \mid \quad 1 \leq i \leq q-1\right\}$ is exactly the set of degree 2 covers $Z \rightarrow \mathbb{P}_{y}^{1}$ which are quotients of $S_{m} \rightarrow \mathbb{P}_{y}^{1}$. By [6, Proposition 3], an application of [9, Theorem C], there is an isogeny

$$
\operatorname{Jac}\left(S_{m}\right) \sim \bigoplus_{i=1}^{q-1} \operatorname{Jac}\left(Z_{i}\right) .
$$

By Artin-Schreier theory, $\mu_{i} f(y)$ can be modified by any polynomial of the form $T^{2}-T$ for $T \in \overline{\mathbb{F}}_{2}[y]$ without changing the $\overline{\mathbb{F}}_{2}$-isomorphism class of the Artin-Schreier cover $Z_{i} \rightarrow \mathbb{P}_{y}^{1}$. Thus $Z_{i}$ is isomorphic to an Artin-Schreier curve with equation $z_{i}^{2}-z_{i}=h_{i}(y)$ for some $h_{i}(y) \in \overline{\mathbb{F}}_{2}[y]$ with degree $2 q_{0}+1=$ $\max \left\{\left(q_{0}+q\right) / q_{0}, q_{0}+1\right\}$. For $1 \leq i \leq q-1$, the curve $Z_{i}$ is a $\mathbb{Z} / 2$-cover of the projective line branched only at $\infty$, where it is totally ramified. Moreover, the break in the filtration of higher ramification groups in the lower numbering is at index $\operatorname{deg}\left(h_{i}(y)\right)=2 q_{0}+1$. By [14, VI.4.1], the genus of $Z_{i}$ is $q_{0}$. Thus $g=\operatorname{dim}\left(\operatorname{Jac}\left(S_{m}\right)\right)=(q-1) \operatorname{dim}\left(\operatorname{Jac}\left(Z_{i}\right)\right)=q_{0}(q-1)$.

Remark 3.2. Consider the Artin-Schreier curve $Z_{i}: z_{i}^{2}-z_{i}=h_{i}(y)$ from the proof of Lemma 3.1. By [1, Proposition 3.4], since $\operatorname{deg}\left(h_{i}\right)=2 q_{0}+1 \equiv 1 \bmod 4$, the $a$-number of $Z_{i}$ is $q_{0} / 2$. Thus the $a$-number of $\bigoplus_{i=1}^{q-1} \operatorname{Jac}\left(Z_{i}\right)$ is $q_{0}(q-1) / 2$, exactly half of the genus of $S_{m}$. The fact that $\operatorname{Jac}\left(S_{m}\right)$ is isogenous to $\bigoplus_{i=1}^{q-1} \operatorname{Jac}\left(Z_{i}\right)$ gives little information about the $a$-number of $S_{m}$ since the $a$-number is not an isogeny invariant.

The Hasse-Weil bound states that a (smooth, projective, connected) curve $X$ of genus $g$ defined over $\mathbb{F}_{q}$ must satisfy

$$
q+1-2 g \sqrt{q} \leq \# X\left(\mathbb{F}_{q}\right) \leq q+1+2 g \sqrt{q} .
$$

A curve that meets the upper bound is called an $\mathbb{F}_{q}$-maximal curve.

The number of $\mathbb{F}_{q}$-points on the Suzuki curve is $\# S_{m}\left(\mathbb{F}_{q}\right)=q^{2}+1$. This means that $S_{m}$ is not maximal over $\mathbb{F}_{q}$, but it does have the maximal number of $\mathbb{F}_{q^{-}}$ points possible for a curve of its genus [8, Proposition 2.1]. Analyzing powers of the eigenvalues of Frobenius shows the following.

Lemma 3.3. The Suzuki curve $S_{m}$ is $\mathbb{F}_{q^{4}}$-maximal.

Proof. The $L$-polynomial of $S_{m}$ is $L\left(S_{m}, t\right)=\left(1+\sqrt{2 q} t+q t^{2}\right)^{g}$ [7, Proposition 4.3]. This factors as $L\left(S_{m}, t\right)=(1-\alpha t)^{g}(1-\bar{\alpha} t)^{g}$, where $\alpha=2 q_{0}(1+i)$. That implies that $\# S_{m}\left(\mathbb{F}_{q^{4}}\right)=q^{4}+1-\left(\alpha^{4}+\bar{\alpha}^{4}\right) g=q^{4}+1+2 q^{2} g$, which shows that $S_{m}$ is $\mathbb{F}_{q^{4} \text {-maximal. }}$

A curve which is maximal over a finite field is supersingular in that the slopes of the Newton polygon of its $L$-polynomial all equal $1 / 2$. Thus $S_{m}$ is supersingular. The supersingularity condition is equivalent to the condition that $\operatorname{Jac}\left(S_{m}\right)$ is 
isogenous to a product of supersingular elliptic curves. A supersingular curve in characteristic 2 has 2-rank 0 . This implies, a priori, that the $a$-number of $S_{m}$ is at least one.

3.2. Regular 1-forms. To compute a basis for the vector space $H^{0}\left(S_{m}, \Omega^{1}\right)$ of regular 1-forms on $S_{m}$, consider the functions $h_{1}, h_{2} \in \mathbb{F}\left(S_{m}\right)$ given by

$$
\begin{aligned}
& h_{1}:=z^{2 q_{0}}+y^{2 q_{0}+1}, \\
& h_{2}:=z^{2 q_{0}} y+h_{1}^{2 q_{0}} .
\end{aligned}
$$

For any $f \in \mathbb{F}\left(S_{m}\right)$, let $v_{\infty}(f)$ denote the valuation of $f$ at $P_{\infty}$.

Lemma 3.4. The functions $y, z, h_{1}, h_{2} \in \mathbb{F}\left(S_{m}\right)$ have no poles except at $P_{\infty}$ where

$$
\begin{aligned}
v_{y} & :=-v_{\infty}(y)=q, & v_{z}: & =-v_{\infty}(z)=q+q_{0}, \\
v_{h_{1}}: & =-v_{\infty}\left(h_{1}\right)=q+2 q_{0}, & v_{h_{2}}: & =-v_{\infty}\left(h_{2}\right)=q+2 q_{0}+1 .
\end{aligned}
$$

The function $\pi=h_{1} / h_{2}$ is a uniformizer at $P_{\infty}$.

Proof. See [8, Lemma 1.8].

The function $y$ is a separating variable so $d y$ is a basis of the 1-dimensional vector space of 1 -forms. The next lemma shows that $d y$ is regular.

Lemma 3.5. The 1-form dy satisfies

$$
v_{\infty}(d y)=2 g-2 \quad \text { and } \quad v_{P}(d y)=0
$$

for all points $P \in S_{m}\left(\overline{\mathbb{F}}_{q}\right)$.

Proof. Recall that $\pi$ is a uniformizer at $P_{\infty}$. To take the valuation of $d y$ at $P_{\infty}$, we first rewrite $d y=f(x, y) d \pi$ for some $f(y, z) \in \mathbb{F}_{q}(y, z)$. Note that

$$
d \pi=d\left(\frac{h_{1}}{h_{2}}\right)=\frac{h_{2} d h_{1}-h_{1} d h_{2}}{h_{2}^{2}}=\frac{h_{2} y^{2 q_{0}}-h_{1} z^{2 q_{0}}}{h_{2}^{2}} d y .
$$

Since $v_{\infty}\left(h_{2}^{2}\right)=-2\left(q+2 q_{0}+1\right)$ and

$$
\begin{aligned}
v_{\infty}\left(h_{2} y^{2 q_{0}}-h_{1} z^{2 q_{0}}\right) & =\min \left\{-2 q_{0} v_{y}-v_{h_{2}},-2 q_{0} v_{z}-v_{h_{1}}\right\} \\
& =-2 q_{0} v_{z}-v_{h_{1}} \\
& =-4 q_{0}^{3}-4 q_{0}^{2}-2 q_{0},
\end{aligned}
$$

we see that

$$
\begin{aligned}
v_{\infty}(d y) & =v_{\infty}\left(\frac{h_{2}^{2}}{h_{2} y^{2 q_{0}}-h_{1} z^{2 q_{0}}} d \pi\right) \\
& =-2 q-4 q_{0}-2-\left(-4 q_{0}^{3}-4 q_{0}^{2}-2 q_{0}\right) \\
& =4 q_{o}^{3}-2 q_{0}-2 \\
& =2 g-2 .
\end{aligned}
$$

We next show that $d y$ has no zero or pole at any affine point of $S_{m}$. Note that, for any $a \in \overline{\mathbb{F}}_{q}$, the polynomial $z^{q}+z+a$ splits into distinct factors in $\overline{\mathbb{F}}_{q}(z)$, so there are exactly $q$ points of $S_{m}\left(\overline{\mathbb{F}}_{q}\right)$ lying over any $y_{0} \in \mathbb{A}_{y}^{1}\left(\overline{\mathbb{F}}_{q}\right)$. Since

$$
\left[\overline{\mathbb{F}}_{q}(y, z): \overline{\mathbb{F}}_{q}(y)\right]=q,
$$


the $\overline{\mathbb{F}}_{q}$-Galois cover $S_{m} \rightarrow \mathbb{P}_{y}^{1}$ is unramified at all affine points of $S_{m}$. Consequently, for any point $P \in S_{m}\left(\overline{\mathbb{F}}_{q}\right)$ lying over $a \in \mathbb{A}_{y}^{1}\left(\overline{\mathbb{F}}_{q}\right)$, we see that $v_{P}(y-a)=1$. Thus, $y-a$ is a uniformizer at $P$ and $v_{P}(d y)=0$, proving the proposition.

By Lemma 3.5 finding a basis for $H^{0}\left(S_{m}, \Omega^{1}\right)$ is equivalent to finding a basis for $L((d y))$, since $(d y)=(2 g-2) P_{\infty}$ is the canonical divisor. To do this, we make use of the relations

$$
z^{2}=y h_{1}+h_{2}, \quad h_{1}^{q_{0}}=z+y^{q_{0}+1}, \quad h_{2}^{q_{0}}=h_{1}+z y^{q_{0}},
$$

which can be verified by direct substitution and the following proposition.

Proposition 3.6 ([8, Proposition 1.5]). Let $S G$ be the semigroup

$$
\left\langle q, q+q_{0}, q+2 q_{0}, q+2 q_{0}+1\right\rangle .
$$

Then \# $\{n \in S G \mid 0 \leq n \leq 2 g-2\}=g$.

We now have all the required information to find a basis of $H^{0}\left(S_{m}, \Omega^{1}\right)$.

Proposition 3.7. The following set is a basis of $H^{0}\left(S_{m}, \Omega^{1}\right)$ :

$$
\mathcal{B}:=\left\{y^{a} z^{b} h_{1}^{c} h_{2}^{d} d y \mid(a, b, c, d) \in \mathcal{E}\right\},
$$

where $\mathcal{E}$ is the set of $(a, b, c, d) \subset \mathbb{Z}^{4}$ satisfying

$$
\begin{gathered}
0 \leq b \leq 1, \quad 0 \leq c \leq q_{0}-1, \quad 0 \leq d \leq q_{0}-1, \\
a v_{y}+b v_{z}+c v_{h_{1}}+d v_{h_{2}} \leq 2 g-2 .
\end{gathered}
$$

Proof. To prove linear independence, it suffices to prove that all elements in our basis have distinct valuations at $P_{\infty}$. Suppose $y^{a} z^{b} h_{1}^{c} h_{2}^{d} d y \in \mathcal{B}$ and $y^{a^{\prime}} z^{b^{\prime}} h_{1}^{c^{\prime}} h_{2}^{d^{\prime}} d y$ $\in \mathcal{B}$ have the same valuation at $P_{\infty}$; we will show they are equal. Comparing their valuations at $P_{\infty}$, we must have that

$$
\left(a-a^{\prime}\right) v_{y}+\left(b-b^{\prime}\right) v_{z}+\left(c-c^{\prime}\right) v_{h_{1}}+\left(d-d^{\prime}\right) v_{h_{2}}=0 .
$$

Now consider Equation (3.3) modulo $q_{0}$. As $q_{0}$ divides $v_{y}, v_{z}$ and $v_{h_{1}}$,

$$
\left(d-d^{\prime}\right) \equiv 0 \bmod q_{0} .
$$

As $0 \leq d, d^{\prime}<q_{0}$, it must be the case that $d=d^{\prime}$. Substituting $d-d^{\prime}=0$ into Equation (3.3) and reducing modulo $2 q_{0}$ yields that

$$
\left(b-b^{\prime}\right) q_{0} \equiv 0 \bmod 2 q_{0} .
$$

However, as $0 \leq b, b^{\prime} \leq 1$, it must also be the case that $b=b^{\prime}$. Simplifying (3.3) and reducing modulo $q=2 q_{o}^{2}$ yields that

$$
\left(c-c^{\prime}\right)\left(q-2 q_{0}\right)=\left(c-c^{\prime}\right) 2 q_{0} \equiv 0 \bmod 2 q_{0}^{2} .
$$

Since $0 \leq c, c^{\prime} \leq q_{0}-1$, we find that $c=c^{\prime}$, so $a=a^{\prime}$ as well.

We claim that the above set also spans $L((d y))$. Clearly the valuations at $P_{\infty}$ of

$$
\left\{y^{a} z^{b} h_{1}^{c} h_{2}^{c} \mid a v_{y}+b v_{z}+c v_{h_{1}}+d v_{h_{2}} \leq 2 g-2\right\}
$$

are equal to $\{n \in S G \mid 0 \leq n \leq 2 g-2\}$, which is a set of size $g$ by Proposition 3.6. Rewriting elements of the above set in terms of our basis will not change their valuation at $P_{\infty}$. Thus we can use the relations in Equation (3.2) to see that $\mathcal{B}$ also contains an element for each of the $g$ possible valuations at $P_{\infty}$. By the previous paragraphs, each valuation occurs exactly once. By Riemann-Roch, $\ell((d y))=g$, so $\mathcal{B}$ is a basis. 
3.3. Action of the Cartier operator. The (modified) Cartier operator $\mathcal{C}$ is the semi-linear map $\mathcal{C}: H^{0}\left(S_{m}, \Omega^{1}\right) \rightarrow H^{0}\left(S_{m}, \Omega^{1}\right)$ which annihilates exact 1-forms, preserves logarithmic ones, and is $p^{-1}$-linear. In characteristic 2 , the Cartier operator $\mathcal{C}$ acts on 1 -forms according to the following properties (see, e.g., [15, Section 2.2.5]):

(1) $\mathcal{C}$ is $1 / 2$-linear; i.e., $\mathcal{C}$ is additive and $\mathcal{C}\left(f^{2} \omega\right)=f \mathcal{C}(\omega)$.

(2) $\mathcal{C}\left(y^{j} d y\right)= \begin{cases}0 & \text { if } j \equiv 0 \bmod 2, \\ y^{e-1} d y & \text { if } j=2 e-1\end{cases}$

(3) $\mathcal{C}(\omega)=0$ if and only if $\omega$ is exact, i.e., if and only if $\omega=d f$ for some $f \in \mathbb{F}_{q}\left(S_{m}\right)$.

(4) $\mathcal{C}(\omega)=\omega$ if and only if $\omega=d f / f$ for some $f \in \mathbb{F}_{q}\left(S_{m}\right)$.

Any 1-form $\omega \in H^{0}\left(S_{m}, \Omega^{1}\right)$ can be written in the form $\omega=\left(f^{2}+g^{2} y\right) d y$ as $\operatorname{char}\left(\mathbb{F}_{q}\right)=2$. Then

$$
\mathcal{C}\left(\left(f^{2}+g^{2} y\right) d y\right)=g d y .
$$

By these properties, it is clear that

$$
\mathcal{C}\left(y^{2 e_{1}+r_{1}} z^{2 e_{2}+r_{2}} h_{1}^{2 e_{3}+r_{3}} h_{2}^{2 e_{4}+r_{4}} d y\right)=y^{e_{1}} z^{e_{2}} h_{1}^{e_{3}} h_{2}^{e_{4}} \mathcal{C}\left(y^{r_{1}} z^{r_{2}} h_{1}^{r_{3}} h_{2}^{r_{4}} d y\right) .
$$

Hence to compute the action of $\mathcal{C}$ on $H^{0}\left(S_{m}, \Omega^{1}\right)$, we need only compute $\mathcal{C}$ on the 16 monomials in $y, z, h_{1}, h_{2}$ of degree less than or equal to one in each variable. Table 1 shows this action, where each $\mathcal{C}(f d y)$ is written in terms of the original basis using the curve Equation (3.1).

TABLE 1

\begin{tabular}{|l|l|}
\hline$f$ & $\mathcal{C}(f d y)$ \\
\hline \hline 1 & 0 \\
\hline$y$ & $d y$ \\
\hline$z$ & $y^{q_{0} / 2} d y$ \\
\hline$h_{1}$ & $y^{q_{0}} d y$ \\
\hline$h_{2}$ & $\left(\left(y h_{1}\right)^{q_{0} / 2}+h_{2}^{q_{0}}\right) d y$ \\
\hline$y z$ & $h_{1}^{q_{0} / 2} d y$ \\
\hline$y h_{1}$ & $\left(\left(y h_{1}\right)^{q_{0} / 2}+h_{2}^{q_{0}}\right) d y$ \\
\hline$z h_{1}$ & $\left(y h_{2}\right)^{q_{0} / 2} d y$ \\
\hline$z h_{2}$ & $\left(h_{1} h_{2}\right)^{q_{0} / 2} d y$ \\
\hline$h_{1} h_{2}$ & $\left(h_{1}+z y^{q_{0}}\right) d y$ \\
\hline$y z h_{1}$ & $\left(y^{q_{0} / 2} z+\left(h_{1} h_{2}\right)^{q_{0} / 2}\right) d y$ \\
\hline$y z h_{2}$ & $\left(z h_{1}^{q_{0} / 2}+y^{q_{0} / 2+1} h_{2}^{q_{0} / 2}\right) d y$ \\
\hline$z h_{1} h_{2}$ & $\left(z y^{q_{0} / 2} h_{2}^{q_{0} / 2}+h_{1}^{q_{0} / 2+1}\right) d y$ \\
\hline$y h_{1} h_{2}$ & $\left(\left(y h_{1}\right)^{q_{0} / 2} z+h_{2}^{q_{0} / 2} z\right) d y$ \\
\hline$y z h_{1} h_{2}$ & $\left(y^{q_{0} / 2} h_{2}+z h_{1}^{q_{0} / 2} h_{2}^{q_{0} / 2}\right) d y$ \\
\hline
\end{tabular}


Example 3.8. We illustrate the computation for $z h_{1} h_{2} d y$. Direct computation yields

$$
\begin{aligned}
\mathcal{C}\left(z h_{1} h_{2} d y\right) & =\mathcal{C}\left(z h_{1}\left(y z^{2 q_{0}}+h_{1}^{2 q_{0}}\right) d y\right) \\
& =z^{q_{0}} \mathcal{C}\left(z y h_{1} d y\right)+h_{1}^{q_{0}} \mathcal{C}\left(z h_{1} d y\right) \\
& =\left(y^{q_{0} / 2} z^{q_{0}+1}+h_{1}^{q_{0} / 2} h_{2}^{q_{0} / 2} z^{q_{0}}+h_{1}^{q_{0}} y^{q_{0} / 2} h_{2}^{q_{0} / 2}\right) d y .
\end{aligned}
$$

To write this expression in terms of the original basis, we identify the monomials with the highest pole order at infinity. Since

$$
v_{\infty}\left(h_{1}^{q_{0} / 2} h_{2}^{q_{0} / 2} z^{q_{0}}\right)=v_{\infty}\left(h_{1}^{q_{0}} y^{q_{0} / 2} h_{2}^{q_{0} / 2}\right)=-4 q_{0}^{3}-3 q_{0}^{2}-q_{0} / 2<-(2 g-2),
$$

these two terms may be simplified. Using Equation (3.2),

$$
\begin{aligned}
h_{1}^{q_{0} / 2} h_{2}^{q_{0} / 2} z^{q_{0}}+ & h_{1}^{q_{0}} y^{q_{0} / 2} h_{2}^{q_{0} / 2} \\
& =h_{1}^{q_{0} / 2} h_{2}^{q_{0} / 2}\left(y^{q_{0} / 2} h_{1}^{q_{0} / 2}+h_{2}^{q_{0} / 2}\right)+y^{q_{0} / 2} h_{1}^{q_{0}} h_{2}^{q_{0} / 2}=h_{1}^{q_{0} / 2} h_{2}^{q_{0}} .
\end{aligned}
$$

The final expression follows by rewriting $z^{q_{0}+1}$ and $h_{2}^{q_{0}}$ in terms of lower order basis elements using Equation (3.2).

Remark 3.9. To compute $\mathcal{C}(\omega)$ for a general element $\omega \in \mathcal{B}$, simply apply Equation (3.5) and use the table above. In nearly all cases the direct result will again be in terms of the basis $\mathcal{B}$. The only exception is when $\omega=z h_{1}^{q_{0}-1} h_{2} d y$. In this case we have

$$
\begin{aligned}
\mathcal{C}\left(h_{1}^{q_{0}-2} \cdot z h_{1} h_{2} d y\right) & =h_{1}^{q_{0} / 2-1} \mathcal{C}\left(z h_{1} h_{2}\right) \\
& =\left(z y^{q_{0} / 2} h_{1}^{q_{0} / 2-1} h_{2}^{q_{0} / 2}+h_{1}^{q_{0}}\right) d y .
\end{aligned}
$$

Using Equation (3.2), one can obtain an expression in terms of the original basis.

\section{The $a$-NUMBer FOR SUZUKI CURVES}

We now have the tools to compute a closed form formula for the $a$-number $a(m)$. The calculation amounts to counting lattice points in polytopes in $\mathbb{R}^{3}$, which is a hard problem in general. In our case, however, the values $v_{y}, v_{h_{1}}$, and $v_{h_{2}}$ are so similar that the polytopes in question are nearly regular; this makes our counting problem much easier.

Theorem 4.1. Let $a(m)$ and $g(m)$ be the a-number and genus of $S_{m}$, respectively. Then

In particular,

$$
a(m)=\frac{q_{0}\left(q_{0}+1\right)\left(2 q_{0}+1\right)}{6} .
$$

$$
\frac{1}{6}<\frac{a(m)}{g(m)}<\frac{1}{6}+\frac{1}{2^{m+1}} .
$$

Proof. Recall from Section 2 that $a(m)$ is the dimension of the kernel of $\mathcal{C}$ on $H^{0}\left(S_{m}, \Omega^{1}\right)$. By Equation (3.4),$a(m)$ is the dimension of the vector space of regular 1 -forms of the form $f^{2} d y$. Since $f^{2}$ can have a pole only at $P_{\infty}$, and since the order of the pole can be at most $2 g-2$, we see that $a(m)=\ell\left((g-1) P_{\infty}\right)$. Moreover, squaring is a homomorphism, so

$$
\ell\left((g-1) P_{\infty}\right)=\#\left\{\omega \in \mathcal{B} \mid v_{\infty}(\omega) \leq g-1\right\} .
$$


By Section 3, this number is exactly

$$
\#\left\{(a, b, c, d) \in \mathcal{E} \mid v_{y} a+v_{z} b+v_{h_{1}} c+v_{h_{2}} d \leq g-1\right\} .
$$

Here we use the notation $\mathcal{E}$ as we did in Proposition 3.7 Recall that $b \in\{0,1\}$. When $b=0$, we must count $\left\{(a, c, d) \in \mathbb{N}^{3} \mid a+c+d \leq q_{0}-1\right\}$. This follows from the fact that

$$
q_{0}-1=\frac{g-1}{v_{h_{2}}}<\frac{g-1}{v_{h_{1}}}<\frac{g-1}{v_{y}}<q_{0} .
$$

For $b=1$, we must count $\left\{(a, c, d) \in \mathbb{N}^{3} \mid a+c+d \leq q_{0}-2\right\}$ since

$$
q_{0}-2<\frac{g-1-v_{z}}{v_{h_{2}}}<\frac{g-1-v_{z}}{v_{h_{1}}}<\frac{g-1-v_{z}}{v_{y}}<q_{0}-1 .
$$

Using these two facts, we obtain

$$
\begin{aligned}
a(m)= & \#\left\{(a, c, d) \in \mathbb{N}^{3} \mid a+c+d \leq q_{0}-1\right\} \\
& +\#\left\{(a, c, d) \in \mathbb{N}^{3} \mid a+c+d \leq q_{0}-2\right\} \\
= & \sum_{i=2}^{q_{0}+1}\left(\begin{array}{l}
i \\
2
\end{array}\right)+\sum_{i=2}^{q_{0}}\left(\begin{array}{l}
i \\
2
\end{array}\right) \\
= & 1+\sum_{i=2}^{q_{0}}\left(\left(\begin{array}{c}
i+1 \\
2
\end{array}\right)+\left(\begin{array}{l}
i \\
2
\end{array}\right)\right) \\
= & \sum_{i=1}^{q_{0}} i^{2},
\end{aligned}
$$

which equals the formula in the first statement.

To prove the second statement, simply note that

$$
\frac{1}{6}<\frac{q_{0}\left(q_{0}+1\right)\left(2 q_{0}+1\right)}{6 q_{0}(q-1)}=\frac{1}{6} \cdot \frac{q_{0}^{2}+\frac{3}{2} q_{0}+\frac{1}{2}}{q_{0}^{2}-\frac{1}{2}}<\frac{1}{6}\left(1+\frac{3}{q_{0}}\right) .
$$

\section{Open QUeSTIONS}

Here are two open questions about $\operatorname{Jac}\left(S_{m}\right)$.

Question 5.1. What is the decomposition of $\operatorname{Jac}\left(S_{m}\right)$ into indecomposable principally polarized abelian varieties?

Theorem 4.1 gives partial information about Question 5.1, namely an upper bound on the number of factors appearing in the decomposition, because of the following fact.

Lemma 5.2. Suppose $A$ is a principally polarized abelian variety with $p$-rank 0 and $a$-number a. If $A$ decomposes as the direct sum of $t$ principally polarized abelian varieties, then $t \leq a$.

Proof. Write $A \simeq \bigoplus_{i=1}^{t} A_{i}$, where each $A_{i}$ is a principally polarized abelian variety. For $1 \leq i \leq t$, consider the $p$-torsion group scheme $A_{i}[p]$. The $a$-number of $A_{i}[p]$ is at least 1 since its $p$-rank is 0 . Thus the $a$-number of $A$ is at least $t$. 
To state the second question, we need some more notation.

The Ekedahl-Oort type of a principally polarized abelian variety $A$ over $k$ is defined by the interaction between the Frobenius $F$ and Verschiebung $V$ operators on the $p$-torsion group scheme $A[p]$. It determines the isomorphism class of $A[p]$ and its invariants such as the $a$-number. To define the Ekedahl-Oort type, recall that the isomorphism class of a symmetric $B T_{1}$ group scheme $\mathbb{G}$ over $k$ can be encapsulated into combinatorial data. This topic can be found in 13 . If $\mathbb{G}$ has rank $p^{2 g}$, then there is a final filtration $N_{1} \subset N_{2} \subset \cdots \subset N_{2 g}$ of $\mathbb{G}$ as a $k$-vector space which is stable under the action of $V$ and $F^{-1}$ such that $i=\operatorname{dim}\left(N_{i}\right)$. The Ekedahl-Oort type of $\mathbb{G}$, also called the final type, is $\nu=\left[\nu_{1}, \ldots, \nu_{r}\right]$, where $\nu_{i}=\operatorname{dim}\left(V\left(N_{i}\right)\right)$. The Ekedahl-Oort type of $\mathbb{G}$ is canonical even if the final filtration is not.

There is a restriction $\nu_{i} \leq \nu_{i+1} \leq \nu_{i}+1$ on the final type. Moreover, all sequences satisfying this restriction occur. This implies that there are $2^{g}$ isomorphism classes of symmetric $B T_{1}$ group schemes of rank $p^{2 g}$. The $p$-rank is $\max \left\{i \mid \nu_{i}=i\right\}$, and the $a$-number equals $g-\nu_{g}$.

Question 5.3. What is the Ekedahl-Oort type of $\operatorname{Jac}\left(S_{m}\right)[2]$ ? Equivalently, what is the covariant Dieudonné module of $\operatorname{Jac}\left(S_{m}\right)[2]$ ?

Theorem 4.1 gives partial information about Question 5.3 by limiting the possible final types. For the group scheme Jac $\left(S_{m}\right)$ [2], the Ekedahl-Oort type satisfies that $\nu_{1}=0$ and $\nu_{g}=q_{0}\left(10 q_{0}+7\right)\left(q_{0}-1\right) / 6$. In particular, $\operatorname{Jac}\left(S_{m}\right)$ is not superspecial since $a(m) \neq g(m)$. This implies that $\operatorname{Jac}\left(S_{m}\right)$ is not isomorphic to the product of supersingular elliptic curves; it is only isogenous to the product of supersingular elliptic curves.

In the next example, we give some more information about the Ekedahl-Oort type of $\operatorname{Jac}\left(S_{1}\right)[2]$ (the case $m=1$ ).

Example 5.4. If $m=1$, then $q_{0}=2, q=8$, and $g=14$. By Section 3.3, the image of $\mathcal{C}$ on $H^{0}\left(S_{m}, \Omega^{1}\right)$ is spanned by the nine 1 -forms

$$
\left\{d y, y d y, h_{1} d y, y^{2} d y, y h_{1} d y, y h_{2},\left(z+y^{3}\right) d y, h_{1} h_{2} d y, y^{2} z d y\right\} .
$$

The image of $\mathcal{C}^{2}$ on $H^{0}\left(S_{m}, \Omega^{1}\right)$ is spanned by the four 1 -forms

$$
\left\{d y, y^{2} d y,\left(z+y^{3}\right) d y,\left(h_{1}+y^{2} z\right) d y\right\} .
$$

Also, $\mathcal{C}^{3}$ trivializes $H^{0}\left(S_{m}, \Omega^{1}\right)$. Thus $\nu_{1}=\nu_{2}=\nu_{3}=\nu_{4}=0, \nu_{9}=4$, and $\nu_{14}=9$. The combinatorial restrictions on the final type imply that $\nu_{10}=5, \nu_{11}=6$, $\nu_{12}=7$, and $\nu_{13}=8$. This leaves only five possibilities for the final type and thus for the isomorphism class of $\operatorname{Jac}\left(S_{1}\right)[2]$.

\section{ACKNOWLEDGEMENTS}

The authors would like to thank the NSF for sponsoring the research workshop for graduate students at Colorado State University in June 2011, where the work on this project was initiated. The authors would also like to thank Amy Ksir and the other workshop participants for their insights and the referee for the quick report and helpful comments. 


\section{REFERENCES}

1. Arsen Elkin and Rachel Pries, Ekedahl-Oort strata of hyperelliptic curves in characteristic 2, arXiv:1007.1226. To appear in Algebra and Number Theory.

2. Rainer Fuhrmann and Fernando Torres, On Weierstrass points and optimal curves, Rend. Circ. Mat. Palermo (2) Suppl. (1998), no. 51, 25-46. MR.1631013 (99e:11081)

3. Arnaldo García and Henning Stichtenoth, Elementary abelian p-extensions of algebraic function fields, Manuscripta Math. 72 (1991), no. 1, 67-79. MR1107453 (92j:11139)

4. Massimo Giulietti and Gábor Korchmáros, On automorphism groups of certain Goppa codes, Des. Codes Cryptogr. 47 (2008), no. 1-3, 177-190. MR2375466 (2009d:94156)

5. Massimo Giulietti, Gábor Korchmáros, and Fernando Torres, Quotient curves of the Suzuki curve, Acta Arith. 122 (2006), no. 3, 245-274. MR2239917 (2007g:11069)

6. Darren Glass and Rachel Pries, Hyperelliptic curves with prescribed p-torsion, Manuscripta Math. 117 (2005), no. 3, 299-317. MR.2154252 (2006e:14039)

7. Johan P. Hansen, Deligne-Lusztig varieties and group codes, Coding theory and algebraic geometry (Luminy, 1991), Lecture Notes in Math., vol. 1518, Springer, Berlin, 1992, pp. 6381. MR.1186416 (94e:94024)

8. Johan P. Hansen and Henning Stichtenoth, Group codes on certain algebraic curves with many rational points, Appl. Algebra Engrg. Comm. Comput. 1 (1990), no. 1, 67-77. MR.1325513 (96e:94023)

9. E. Kani and M. Rosen, Idempotent relations and factors of Jacobians, Math. Ann. 284 (1989), no. 2, 307-327. MR1000113 (90h:14057)

10. H. Kraft, Kommutative algebraische p-gruppen (mit anwendungen auf p-divisible gruppen und abelsche varietäten), manuscript, University of Bonn, September 1975, 86 pp.

11. K.-Z. Li and F. Oort, Moduli of supersingular abelian varieties, Lecture Notes in Mathematics, vol. 1680, Springer-Verlag, Berlin, 1998. MR1611305 (99e:14052)

12. B. Moonen, Group schemes with additional structures and Weyl group cosets, Moduli of abelian varieties (Texel Island, 1999), Progr. Math., vol. 195, Birkhäuser, Basel, 2001, pp. 255298. MR,1827024 (2002c:14074)

13. F. Oort, A stratification of a moduli space of abelian varieties, Moduli of abelian varieties (Texel Island, 1999), Progr. Math., vol. 195, Birkhäuser, Basel, 2001, pp. 345-416. MR2002b:14055

14. Henning Stichtenoth, Algebraic function fields and codes, second ed., Graduate Texts in Mathematics, vol. 254, Springer-Verlag, Berlin, 2009. MR2464941(2010d:14034)

15. Michael Tsfasman, Serge Vlădut, and Dmitry Nogin, Algebraic geometric codes: basic notions, Mathematical Surveys and Monographs, vol. 139, American Mathematical Society, Providence, RI, 2007. MR2339649(2009a:94055)

Department of Mathematics, University of Massachusetts-Amherst, Amherst, MasSACHUSETTS 01003

E-mail address: holleyf@math.umass .edu

Department of Mathematics, University of Wisconsin-Madison, Madison, Wisconsin 53706

E-mail address: garton@math.wisc.edu

Current address: Department of Mathematics, Northwestern University, Evanston, Illinois 60208

Department of Mathematics, Wesleyan University, Middletown, Connecticut 06457 E-mail address: emalmskog@wesleyan.edu

Current address: Department of Mathematics and Statistics, Colorado College, Colorado Springs, Colorado 80946

Department of Mathematics, Colorado State University, Fort Collins, Colorado 80521

E-mail address: pries@math.colostate.edu

Department of Mathematics and Statistics, University of Calgary, Calgary, AB, CANADA T2N $1 \mathrm{~N} 4$

E-mail address: cjweir@ucalgary.ca 